\title{
Theoretical knowledge and self-assessed ability to perform cardiopulmonary resuscitation: a survey among 3044 healthcare professionals in Sweden
}

\author{
Jennie Silverplats ${ }^{a, b}$, Marie-Louise Södersved Källestedt ${ }^{c}$ \\ Philippe Wagner ${ }^{c}$, Annica Ravn-Fischer ${ }^{\mathrm{d}}$, Björn Äng ${ }^{\mathrm{a}, \mathrm{e}, \mathrm{f}}$ and \\ Anneli Strömsöe $e^{a, f, g}$
}

\begin{abstract}
Objective Theoretical knowledge and ability to perform cardiopulmonary resuscitation (CPR) are unknown with regard to provided training. The aim of this study was to evaluate in-hospital healthcare professionals' (HCPs) theoretical knowledge of CPR and their self-assessed ability to perform CPR and also to assess possible affecting factors.
\end{abstract}

Method A questionnaire was sent to $n=5323 \mathrm{HCPs}$ containing a nine-question knowledge test and a Likert scale measuring self-assessed ability. A factor score of self-assessed ability and a ratio scale of correct answers were dependent variables in multiple linear regression.

Results Only $41 \%$ of the responding HCPs passed the knowledge test with seven or more correct answers. Nurses had the highest pass rate $(50 \%)$ and the highest attendance rate at CPR training (56\%). The ability to perform defibrillation was strongly agreed by $43 \%$ and the ability of leadership by only $7 \%$. Working on a monitored ward, CPR training 0-6 months ago and being a nurse or physician were factors associated with more correct answers and higher ratings of abilities.

\section{Introduction}

Theoretical knowledge of cardiopulmonary resuscitation (CPR) is the foundation for giving correct treatment in a cardiac arrest situation. Inadequate knowledge of CPR was found among Swedish healthcare professionals (HCPs) during implementation of CPR with an automated external defibrillator (AED) [1]. Studies conducted outside Sweden suggest similar results regarding knowledge of CPR [2-4]. It is well known that theoretical knowledge and skills received in CPR training decreases with time and retraining is needed for retention [5]. Other factors that might affect theoretical knowledge of CPR and self-assessed ability to perform CPR are poorly studied.

Supplemental Digital Content is available for this article. Direct URL citations appear in the printed text and are provided in the HTML and PDF versions of this article on the journal's website (www.euro-emergencymed.com)

This is an open-access article distributed under the terms of the Creative Commons Attribution-Non Commercial-No Derivatives License 4.0 (CC-BYNC-ND), where it is permissible to download and share the work provided it is properly cited. The work cannot be changed in any way or used commercially without permission from the journal.
Conclusion The overall theoretical knowledge was poor and ratings of self-assessed abilities to perform CPR were low. Working on a monitored ward, recently attended CPR training and being a nurse or physician were factors associated with higher theoretical knowledge and higher ratings of self-assessed ability to perform CPR. These findings imply prioritisation of CPR training. European Journal of Emergency Medicine XXX: 000-000 Copyright (c) 2020 The Author(s). Published by Wolters Kluwer Health, Inc.

European Journal of Emergency Medicine 2020, XXX:000-000

Keywords: automated external defibrillators, cardiac arrest, cardiopulmonary resuscitation, emergency medicine

${ }^{a}$ School of Education, Health and Social Studies, Dalarna University, Falun, bepartment of Anaesthesiology and Intensive Care, Mora Hospital, Region of Dalarna, Mora, ${ }^{\mathrm{C}}$ Centre for Clinical Research Västmanland, Uppsala University, Västerås, ${ }^{\mathrm{d}}$ Department of Cardiology, Sahlgrenska University Hospital, Göteborg, ${ }^{e}$ Department of Neurobiology, Care Sciences and Society, Division of Physiotherapy, Karolinska Institutet, Huddinge, ${ }^{f}$ Centre for Clinical Research Dalarna, Uppsala University, Falun and ${ }^{9}$ Department of Prehospital Care, Region of Dalarna, Falun, Sweden

Correspondence to Jennie Silverplats, RN, Department of Anaesthesiology and Intensive Care, Mora Hospital, Lasarettsvägen 35, S-792 85 Mora, Sweden Tel: +46 250 493305; fax: +46 250 10197; e-mail: jep@du.se

Received 29 October 2019 Accepted 25 February 2020

All in-hospital HCPs involved in patient contact should be able to treat a cardiac arrest with CPR AED according to guidelines from the Swedish Resuscitation Council [6]. From 1998 to 2007, there were 6400 educated in-hospital CPR instructors who in turn had trained 49560 HCPs. Nurses were the largest group of professionals among instructors. In-hospital CPR training programmes consists of CPR AED, introduced into nonmonitored wards (general wards and other in-hospital areas without monitoring equipment) in 2006, and advanced CPR, attended by physicians and nurses on monitored wards since 1989 [7]. CPR training is provided by the hospital employer and includes an online theoretical test combined with peerled practical exercises. The online test can be repeated several times until all questions are correct. CPR training should be repeated regularly - every 6 months or at least once a year [6]. In 2008, $52 \%$ of Swedish hospitals registered their CPR training and $45 \%$ of those had provided training to $75-100 \%$ of all HCPs during the past year [8]. Since then, a national register of CPR training has been 
introduced were all provided training should be registered but the compliance has been low.

The theoretical knowledge of CPR and self-assessed ability to perform CPR are unknown with regard to the current training programmes offered and the amount of training provided among all professions in hospitals today.

The aim of this study was to evaluate in-hospital HCPs' theoretical knowledge of CPR and their self-assessed ability to perform CPR and also to assess possible affecting factors.

\section{Method}

\section{Design and data collection}

The study was conducted as a cross-sectional survey. Data were collected at four secondary-care hospitals in two regions in Sweden. The number of in-patient beds ranged from 45 to 600 with a total catchment area population of $n=543565$. A total of $n=5323$ active-duty HCPs involved in patient contact were included, physicians, nurses (including midwives), nursing assistants (including caretakers and dental nurses), other university-educated health professionals and administrative professions. The HCPs worked on both monitored wards and nonmonitored wards, and at other in-hospital out-patient facilities. Data were collected with questionnaires during the period 2013-2014 in one region during a 5-year follow-up study regarding theoretical CPR knowledge, performed by the second author, and during 2015-2016 in the other region by the first author. Permission was obtained from chief physicians and participation was voluntary and anonymous. HCPs were informed of the study at staff meetings or by e-mail. Questionnaires were distributed and collected at staff-meetings or via mailboxes. The response time ranged from 16 to 25 days.

\section{The questionnaire}

Theoretical knowledge was measured using nine multiple-choice questions addressing basic CPR knowledge. They covered the evaluation of an unconscious patient, chest compressions, mouth-to-mouth ventilation, and defibrillation (Supplemental Digital Content 1, http:// links.lww.com/EJEM/A275 question no. 1-9). Three questions concerned demographic information regarding profession, years in profession and time since CPR training (Supplemental Digital Content 1, http://links.lww. com/EJEM/A275 question no. 12-14). Self-assessed ability was measured using two questions that contained a Likert scale regarding compression, ventilation, defibrillation and leadership. The Likert scale ranged from 1 'I strongly disagree' to 7 'I strongly agree' (Supplemental Digital Content 1, http://links.lww.com/EJEM/A275 question no. 36, $37 \mathrm{a}-\mathrm{c})$. The workplace was noted by the author during the collection of questionnaires.

The nine questions regarding theoretical knowledge were validated by test-retest procedures and interviews [9]. The Likert scale was validated by interviews prior to use.
The questionnaire contained 41 questions, the questions not included in this study will be analysed elsewhere.

\section{Statistical analyses}

The knowledge test results and results of self-assessed abilities were displayed descriptively and evaluated using the following analyses. The passing level of the knowledge test was defined as seven or more correct answers out of nine. Differences between professions regarding knowledge test results and regarding time since CPR training was evaluated using Pearson's Chi-square test. Mann-Whitney's $U$ test was used to compare self-assessed abilities (mean rank of a seven-item Likert scale) between respondents who passed the knowledge test and respondents who did not. A factor score of self-assessed abilities (ranging from -4 to 1.1 ) was created from a Likert scale measuring compression, ventilation, defibrillation and leadership using principal component analysis. The four self-assessed abilities covered the same underlying construct, one component with an eigenvalue of 2.735 representing $68.4 \%$ of the variance and factor loadings ranging from 0.71 to 0.89 . The correlation between self-assessed abilities (factor score) and number of correct answers on the knowledge test (a ratio scale ranging from 0 to 9) was analysed using Spearman's correlation.

Two multiple linear regression analyses were performed for evaluation of affecting factors, one concerning theoretical knowledge and one concerning self-assessed ability. The ratio scale of number of correct answers on the knowledge test (ranging from 0 to 9) was used as a dependent variable in the analysis concerning theoretical knowledge. The factor score (ranging from -4 to 1.1 ) was used as a dependent variable in the analysis concerning self-assessed ability. The independent variables in the two linear regression analyses were profession (a five-item categorical variable), years in profession (a scale of 0-50), workplace (a dichotomous variable of monitored ward or nonmonitored ward) and time since last CPR training (a three-item categorical variable of 0-6 months ago, 7-11 months ago, and 1 year ago or more/ never). An initial Spearman's correlation analysis of the independent variables showed that there was no strong in-between co-linearity $\left(r_{s}<0.6\right)$. All variables and interactions were regressed one by one with the dependent variable in a crude model. All independent variables and interactions associated with the dependent variables at a level of $P<0.05$ were entered into estimating multiple models for analysis displaying unstandardised Beta. Analyses were performed using the IBM Statistical Package for the Social Sciences 25.

\section{Ethical considerations}

The study was given an additional approval by the ethical committee in Uppsala, (Dnr 2006/201/2). The study was registered at ClinicalTrials.gov (ID nr NCT03498508) and was performed according to the principles of the declaration of Helsinki [10]. Consent for publication was not applicable in this study. 


\section{Results}

The response rate was $57 \% \quad(n=3044)$ and internal missing data varied from 0.1 to $3.4 \%$. Table 1 shows the demographics of the respondents including previous CPR training and knowledge test results. Only 3\% $(n=96)$ of the respondents answered all the questions correctly. Forty-one percent $(n=1239)$ passed the knowledge test with seven or more correct answers $(\geq 80 \%)$. Nurses had the highest pass rate and the highest attendance rate in CPR training of all professions (Table 1, and Supplemental Digital Content 2, http://links.lww.com/ EJEM/A276 table with frequencies of correct answers).

A majority of respondents strongly agreed with the ability to perform compressions (62\%) and ventilation (59\%), but the ratings concerning defibrillation and leadership were lower (43 and 7\%) (Fig. 1). A mean of ranks comparison showed higher ratings of abilities among respondents who passed the knowledge test compared to those who did not pass (compression 1654, $1330 P<0.001$, ventilation 1649 , $1334 P<0.001$, defibrillation 1749, $1265 P<0.001$, leadership $1760,1258 P<0.001)$. There was a low positive correlation between the factor score of abilities and number of correct answers, $r_{\mathrm{s}}(2920)=0.39 P<0.001$.

The number of correct answers were significantly lower with longer time since CPR training. Compared to CPR training 0-6 months ago, less correct answers were seen among respondents with CPR training 7-11 months ago (B, -0.398 ; confidence interval (CI), -0.6 to -0.188 ) and 1 year ago or more/never (B, -0.971 ; CI, -1.147 to -0.794 ). Less correct answers were also seen among respondents on nonmonitored wards (B, -0.625 ; CI, -0.814 to $-0.437)$ than among respondents on monitored wards. An interaction showed smaller difference in number of correct answers between nursing assistants on nonmonitored wards versus on monitored wards (B, 0.358; CI, 0.053-0.662). Being a nurse or physician meant more correct answers, with a small insignificant difference to each other, compared to nursing assistants $(\mathrm{B},-0.875$; CI, -1.175 to -0.575$)$, other university-educated staff (B, -0.607 ; CI, -0.904 to -0.311 ) and administrative staff (B, -1.784 ; CI, -2.260 to -1.308 ). Years in profession were not a significant factor regarding number of correct answers and did not fit in the multiple models (Supplemental Digital Content 3, http://links.lww.com/EJEM/A277 factors affecting number of correct answers).

The ratings of abilities were significantly lower with longer time since CPR training. Compared to CPR training 0-6 months ago, lower ratings were seen among respondents with $\mathrm{CPR}$ training $7-11$ months ago $(\mathrm{B}$, -0.184 ; CI, -0.316 to -0.053$)$ and one year ago or more/ never (B, -0.418 ; $\mathrm{CI},-0.529$ to -0.308$)$. An interaction showed a smaller difference in ratings between physicians with CPR training 1 year ago or more/never versus physician with recent CPR training (B, 0.243; CI, 0.015 to 0.471 ). Working on a nonmonitored ward was associated with lower ratings of abilities compared to monitored wards ( $\mathrm{B},-0.235$; $\mathrm{CI},-0.325$ to -0.145 ). Being a nurse or physician meant higher ratings of abilities, with a small insignificant difference to each other, compared to nursing assistants (B, -0.252 ; CI, -0.386 to -0.119$)$, other university-educated staff (B, -0.602 ; CI, -0.786 to -0.418 ) and administrative staff ( $\mathrm{B},-1.337$; $\mathrm{CI},-1.635$ to -1.038 ). Years in profession had little positive effect on ratings of abilities (B, 0.005; CI, 0.003-0.008) (Supplemental Digital Content 4, http://links.lww.com/EJEM/A278 factors affecting self-assessed ability to perform CPR).

\section{Discussion}

The major findings showed that the theoretical knowledge was poor and ratings of self-assessed abilities to perform CPR were low. Working on a monitored ward, recently attended CPR training and being a nurse or physician were factors associated with higher theoretical knowledge and higher ratings of self-assessed ability to perform CPR.

The poor knowledge result in this study is in line with several previous studies [1-4]. This could be because only $49 \%$ of the respondents had attended CPR training during the past year or that provided training is suboptimal. The hospital employer provides peer-led CPR training, which has been proven effective regarding knowledge and skill retention [5]. However, training must be prioritised to

Table 1 Years in profession, time since cardiopulmonary resuscitation training and knowledge test result

\begin{tabular}{|c|c|c|c|c|c|c|c|}
\hline & Physicians & Nurses & Nursing assistants & Other university-educated staff & Administrative staff & Total & Missing \\
\hline & $487(16)$ & $1241(41)$ & $861(28)$ & $337(11)$ & $103(3)$ & 3029 (99) & $15(0.5)$ \\
\hline Years in profession & $14.8(13.8-15.8)$ & $18.3(17.6-18.9)$ & $21.0(20.0-21.9)$ & $16.1(14.9-17.4)$ & $19.2(16.6-21.8)$ & $18.2(17.8-18.7)$ & $68(2)$ \\
\hline Monitored ward & $29(6)$ & $266(21)$ & $163(19)$ & N/A & N/A & $458(15)$ & $0(0)$ \\
\hline \multicolumn{8}{|c|}{$\mathrm{CPR}^{\mathrm{a}}$-training $\leq 11$ months } \\
\hline Monitored ward & $14(48)$ & $171(64)$ & $107(66)$ & N/A & N/A & $292(64)$ & $2(0.4)$ \\
\hline Nonmonitored ward & $154(34)$ & $519(54)$ & $326(47)$ & $158(47)$ & $44(43)$ & $1201(46)$ & $22(0.7)$ \\
\hline All respondents* & $168(35)$ & $690(56)$ & $433(50)$ & $158(47)$ & $44(43)$ & $1493(49)$ & $24(0.8)$ \\
\hline \multicolumn{8}{|l|}{ Knowledge test result } \\
\hline$\geq 80 \%$ correct $^{\star}$ & $195(40)$ & $626(50)$ & 331 (38) & $80(24)$ & $7(7)$ & $1239(41)$ & $0(0)$ \\
\hline $100 \%$ correct & $3(1)$ & $72(6)$ & $19(2)$ & $2(1)$ & $0(0)$ & $96(3)$ & $0(0)$ \\
\hline
\end{tabular}

Respondents are presented as frequencies $n$ and percent (\%), years in profession are presented as mean (95\% confidence interval).

N/A, not applicable.

${ }^{\mathrm{a}}$ Cardiopulmonary resuscitation.

${ }^{\star} P<0.001$. 
Fig. 1

(a)

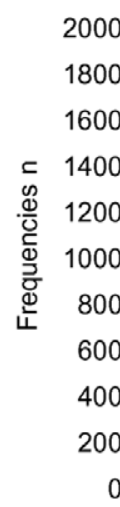

(c)

2000

1800

1600

c 1400

\% 1200

额 1000

Ð 800

600

400

200

0
Compression

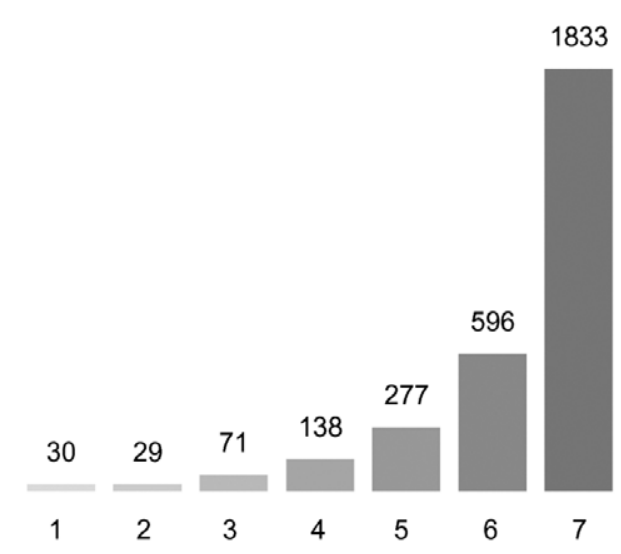

Defibrillation

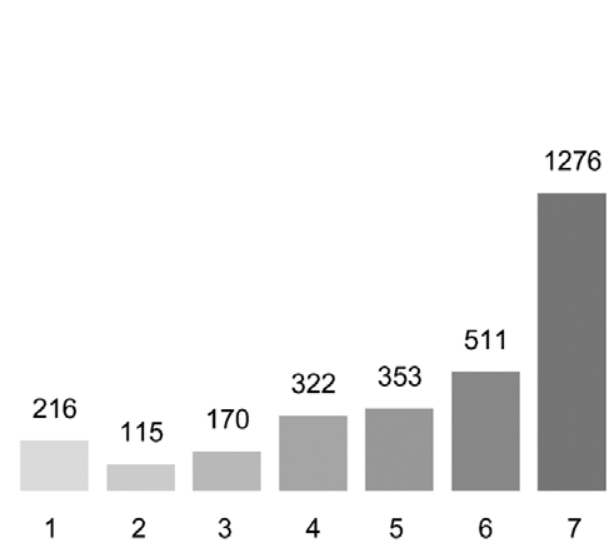

(b)

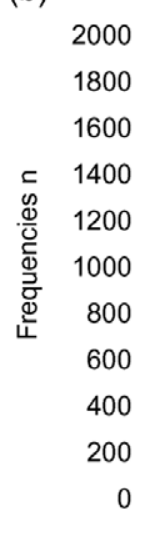

(d)

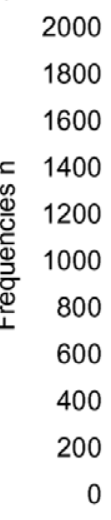

Ventilation

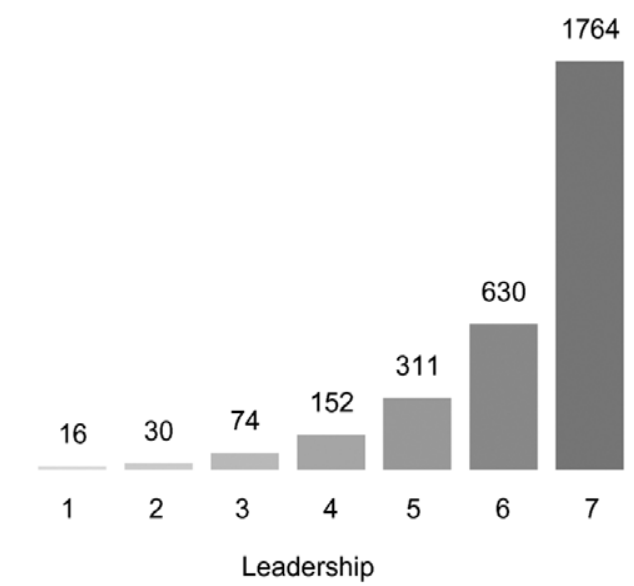

1764

Leadership

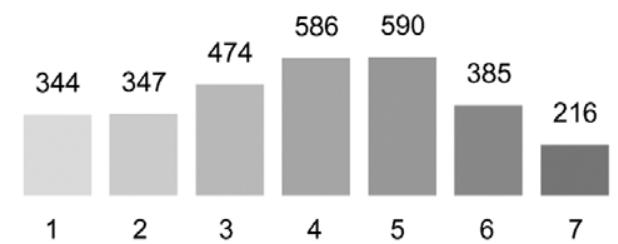

Self-assessed ability to perform CPR rated on a Likert scale ranging from $1=$ 'I strongly disagree' to $7=$ 'I strongly agree'. The abilities consisted of compression (A, internal missing $n=70)$, ventilation (B, internal missing $n=67)$, defibrillation (C, internal missing $n=81)$ and leadership $(D$, internal missing $n=102$ ). Respondents are presented in frequencies $(n)$. CPR, cardiopulmonary resuscitation.

obtain recommended intervals. Other Nordic studies have shown similar results where CPR training was not performed according to recommended intervals [11,12]. The shortage of registered nurses, who often work as CPR instructors, could explain the lack of arranged CPR training. A shortage of nurses leading to increased patient-tonurse ratios could also lead to decreased survival among patients treated for in-hospital cardiac arrest [13].

In this study, only 62 and $59 \%$ of the respondents strongly agreed with the ability to give compressions and ventilations. These treatments, given instantly and correct, are considered most important for patient survival during cardiac arrest [14]. The ratings of defibrillation and leadership were lower which, in theory, could lead to a possible delay of treatment and low team performance. There seems to be a need for team training and simulation with an emphasis on these aspects. Previous leadership training has been found to be the most important factor affecting leadership skills in arrest situations and high leadership skills are associated with better levels of team performance when performing complex skills [15]. In a cardiac arrest situation, someone has to take the lead, which can be passed on when higher medical competence arrives at the scene. Leadership is not only seen as something performed by a member of an emergency team. Self-assessed CPR ability and observed performance on a manikin have been found to correlate well [16]. However, it is not clear if performance on a manikin represents performance in a real cardiac arrest situation.

More correct answers and higher ratings of abilities were seen among respondents on monitored wards and among nurses and physicians. This could be due to a higher incidence rate of cardiac arrest per in-patient bed in monitored wards and experience of cardiac arrests. Experience in cardiac arrest situations seems to be an important factor regarding knowledge and confidence. Five or more cardiac arrest experiences in the previous year have been associated with better knowledge of CPR and HCPs in monitored areas have been found to be more confident with their CPR skills compared to those working 
in nonmonitored areas [2]. HCPs in high-risk areas of cardiac arrest and nursing staff have shown higher motivation towards CPR training and have found CPR skills more important compared to HCPs in low-risk areas and compared to other professions [17]. This might explain the higher attendance rate of CPR training among HCPs on monitored wards and among nurses in this study.

The findings of this study imply a need to prioritise CPR training to obtain recommended training intervals among all professions and workplaces. This study brings awareness to current CPR knowledge and abilities among all groups of in-hospital professions, which have rarely been studied simultaneously before. Resuscitation of a patient with a cardiac arrest is a team effort and the competence of all professions involved in the situation is important for the outcome.

\section{Limitations}

The response rates among professions were equal in one region, but unfortunately not monitored in detail in the other region. Lower response rates were observed on monitored wards in both regions. Nonresponders and missing cases can lead to selection bias with loss of information, which in turn could affect the results. The participants were anonymous and therefore any deeper evaluation of nonresponders could not be made. Physicians on monitored wards were difficult to identify and some of them, therefore, ended up in the nonmonitored group. Although the questions were pretested, the question addressing respondents' self-assessed ability of leadership raises some concerns. It contained both the ability to lead and to give advice to the team, which could have led to misconceptions. The respondents' experience of participating in cardiac arrest situations was not evaluated.

\section{Conclusion}

The overall theoretical knowledge was poor and ratings of self-assessed abilities to perform CPR were low. Working on a monitored ward, recently attended CPR training and being a nurse or physician were factors associated with higher theoretical knowledge and higher ratings of self-assessed ability to perform CPR. These findings imply prioritisation of CPR training.

\section{Acknowledgements}

To the Department of Anaesthesiology and Intensive Care in Mora, for their support and the Centre for Clinical Research Västerås for good collaboration.

\section{Conflicts of interests}

There are no conflicts of interest.

\section{References}

1 Källestedt ML, Rosenblad A, Leppert J, Herlitz J, Enlund M. Hospital employees' theoretical knowledge on what to do in an in-hospital cardiac arrest. Scand J Trauma Resusc Emerg Med 2010; 18:43.

2 Passali C, Pantazopoulos I, Dontas I, Patsaki A, Barouxis D, Troupis $\mathrm{G}$, Xanthos T. Evaluation of nurses' and doctors' knowledge of basic \& advanced life support resuscitation guidelines. Nurse Educ Pract 2011; 11:365-369.

3 Xanthos T, Akrivopoulou A, Pantazopoulos I, Aroni F, Datsis A, lacovidou N. Evaluation of nurses' theoretical knowledge in basic life support: a study in a district Greek hospital. Int Emerg Nurs 2012; 20:28-32.

4 Price CS, Bell SF, Janes SE, Ardagh M. Cardio-pulmonary resuscitation training, knowledge and attitudes of newly-qualified doctors in New Zealand in 2003. Resuscitation 2006; 68:295-299.

5 Greif R, Lockey AS, Conaghan P, Lippert A, De Vries W, Monsieurs KG; Education and implementation of resuscitation section Collaborators; Collaborators. European Resuscitation Council Guidelines for Resuscitation 2015: section 10. Education and implementation of resuscitation. Resuscitation 2015; 95:288-301.

6 Hjärtstopp inom sjukvården - Behandling och utbildning 2017. The Swedish Resuscitation Council; https://www.hlr.nu/wp-content/uploads/2018/03/ Hj\%С3\%A4rtstopp-inom-sjukv\%C3\%A5rden-2017.pdf. [Accessed 16 September 2019].

7 Strömsöe A, Andersson B, Ekström L, Herlitz J, Axelsson A, Göransson KE, et al. Education in cardiopulmonary resuscitation in Sweden and its clinical consequences. Resuscitation 2010; 81:211-216.

8 Aune S, Eldh M, Engdahl J, Holmberg S, Lindqvist J, Svensson L, et al. Improvement in the hospital organisation of CPR training and outcome after cardiac arrest in Sweden during a 10-year period. Resuscitation 2011; 82:431-435.

9 Källestedt ML, Leppert J, Enlund M, Herlitz J. Development of a reliable questionnaire in resuscitation knowledge. Am J Emerg Med 2008; 26:723-728.

10 World Medical Association. World medical association declaration of Helsinki: ethical principles for medical research involving human subjects. JAMA 2013; 310:2191-2194.

11 Hopstock LA. Cardiopulmonary resuscitation; use, training and selfconfidence in skills. A self-report study among hospital personnel. Scand J Trauma Resusc Emerg Med 2008; 16:18.

12 Skrifvars MB, Castrén M, Kurola J, Rosenberg PH. In-hospital cardiopulmonary resuscitation: organization, management and training in hospitals of different levels of care. Acta Anaesthesiol Scand 2002; 46:458-463.

13 McHugh MD, Rochman MF, Sloane DM, Berg RA, Mancini ME, Nadkarni VM, et al.; American Heart Association's Get With The GuidelinesResuscitation Investigators. Better nurse staffing and nurse work environments associated with increased survival of in-hospital cardiac arrest patients. Med Care 2016; 54:74-80.

14 Herlitz J, Bang A, Alsen B, Aune S. Characteristics and outcome among patients suffering from in hospital cardiac arrest in relation to the interval between collapse and start of CPR. Resuscitation 2002; 53:21-27.

15 Yeung JH, Ong GJ, Davies RP, Gao F, Perkins GD. Factors affecting team leadership skills and their relationship with quality of cardiopulmonary resuscitation. Crit Care Med 2012; 40:2617-2621.

16 Lund-Kordahl I, Mathiassen M, Melau J, Olasveengen TM, Sunde K, Fredriksen K. Relationship between level of CPR training, self-reported skills, and actual manikin test performance - an observational study. Int J Emerg Med 2019; 12:2.

17 Hopstock LA. Motivation and adult learning: a survey among hospital personnel attending a CPR course. Resuscitation 2008; 76:425-430. 